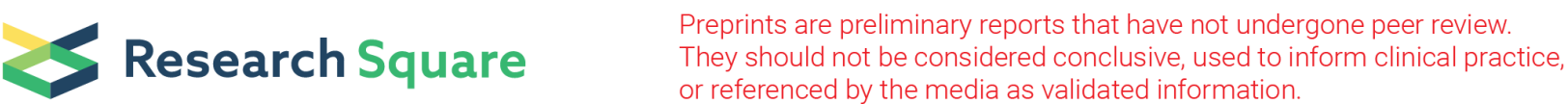

\section{Negative Effects of Acute Appendicitis in Children During COVID-19 Pandemic: Analysis of 1,274 Cases.}

Carlos Delgado-Miguel ( $\square$ carlosdelgado84@hotmail.com )

La Paz Children's Hospital https://orcid.org/0000-0002-4994-6898

Julia Garcia Urbán

Universidad Autónoma de Madrid: Universidad Autonoma de Madrid

Celia Del Monte Ferrer

Universidad Autónoma de Madrid: Universidad Autonoma de Madrid

Antonio Muñoz-Serrano

La Paz University Hospital Children Hospital: Hospital Universitario La Paz Hospital Infantil

\section{María del Carmen Sarmiento}

La Paz University Hospital Children Hospital: Hospital Universitario La Paz Hospital Infantil

\section{Lucas Moratilla}

La Paz University Hospital Children Hospital: Hospital Universitario La Paz Hospital Infantil

\section{Miriam Miguel-Ferrero}

La Paz University Hospital Children Hospital: Hospital Universitario La Paz Hospital Infantil

\section{María De Ceano-Vivas}

La Paz University Hospital Children Hospital: Hospital Universitario La Paz Hospital Infantil

\section{Leopoldo Martínez}

La Paz University Hospital Children Hospital: Hospital Universitario La Paz Hospital Infantil

\section{Research Article}

Keywords: Appendicitis, COVID-19, SARS-CoV-2, Postoperative Complications, Children

Posted Date: September 27th, 2021

DOI: https://doi.org/10.21203/rs.3.rs-930506/v1

License: (c) (i) This work is licensed under a Creative Commons Attribution 4.0 International License. Read Full License 


\section{Abstract}

Introduction: Since the COVID-19 pandemic confinement was established in Spain on March 9, 2020, the number of visits to the paediatric Emergency Department (ED) has decreased dramatically, probably due to the fear of parents becoming infected in the hospital environment. The aim of this work is to analyse the medium-term consequences during the first 9 months after the onset of the COVID-19 pandemic in children with acute appendicitis (AA).

Methods: A retrospective study was performed on children operated on for AA in our institution between 2017-2020, who were distributed in two groups according to the date of surgery: COVID-19 group (after March 9, 2020) and control group (before March 9, 2020). Demographic variables, associated symptoms, time from symptoms onset, hospital stay, rate of complicated AA and postoperative complications were analysed.

Results: A total of 1274 patients were included (288 COVID group; 986 control group), without demographic differences between them. Time from symptom onset was significantly longer in COVID-19 group patients ( 34.5 vs. 24.2 hours; $p=0.021$ ), although no differences in associated symptoms were observed between both groups. COVID-19 group patients presented a higher rate of complicated AA (20.1\% vs. $14 \%$; OR: $1.55 ;$ CI95\%[1.10-2.18]; $p=0.008$ ), a longer hospital stay (3.5 vs. 2.8 days; $p=0.042$ ) as well as a higher rate of postoperative complications (21.5\% vs. $15.7 \%$; OR: 1.47 ; Cl95\%[(1.06-2.04)]; $p=0.008)$.

Conclusion: This study shows the negative medium-term effects of the COVID-19 pandemic on children with acute appendicitis: delayed ED visits, increased rate of complicated AA, increased hospital stay and increased postoperative complications.

\section{Introduction}

When in March 2020 the World Health Organization (WHO) declared the SARS-CoV-2 novel coronavirus (COVID-19) a global pandemic, many Governments implemented a period of mandatory home confinement, colloquially termed as "lockdown", intending to diminish viral transmission (1). This lockdown had a profound impact on visits to the pediatric Emergency Department (ED). In many countries, authorities instructed the population to stay at home and to avoid visiting hospitals as much as possible $(2,3)$. In other cases, parents were extremely concerned with the possibility of their families contracting COVID-19 and were reluctant to take their sick children to medical facilities, even when instructed to do so. Consequently, pediatric ED visits drastically decreased for non-COVID conditions such as dehydration, urinary infection, and acute appendicitis (AA) (4). In pathologies such as AA, this delay in seeking care can lead to increased morbidity and mortality, for longer duration of symptoms prior to consultation is a well-known risk factor for developing complicated appendicitis (5).

In relation to this, several studies have reported a higher rate of complicated appendicitis and postoperative complications in children during the COVID-19 outbreak $(6,7)$, associated with a delay in 
attending the ED. However, most of the studies published have only addressed the acute outbreak COVID19 pandemic lockdown period (March to May 2020), also known as "COVID-19 first wave" $(8,9)$. To date, there are no studies that analyzed the medium-term consequences of the COVID-19 pandemic beyond this first wave. We hypothesized that during the following months after the pandemic outbreak ("COVID19 second wave"), the number of visits to the pediatric ED for suspected AA remained lower than in the previous months, and children were obtaining emergency services later and in more advanced stages of the disease.

The aim of our study was to analyze the negative medium-term effects of AA in children during the first 9 months from the beginning of the pandemic (first and second waves period, from March to December 2020), and to compare these data with a comparable period of time in previous years.

\section{Methods}

A retrospective study was performed on children operated on for AA in our institution between January 2017 and December 2020, who were distributed in two groups according to the date of surgery: COVID-19 group (after March 9, 2020) and control group (before March 9, 2020). Exclusion criteria were age over 16 years, absence of some of the data of the variables analyzed and absence of intraoperative diagnosis of acute appendicitis. Demographic variables, type of abdominal pain (localised or generalised), associated symptoms, time from symptoms onset, mean hospital stay, rate of complicated AA, postoperative complications in the first 30 days after surgery and readmission rate were analysed.

All data were collected from each patient's medical history. Associated symptoms included fever, nausea, vomiting, diarrhoea, constipation, dysuria, anorexia and respiratory symptoms (cough, secretions, stridor). Complicated AA was defined as the presence of peritonitis, perforation or peri-appendicular abscess during surgery. Postoperative complications were defined according to the USA Centers for Disease Control and Prevention's criteria (10). Surgical wound infection, wound dehiscence, intra-abdominal abscess and bowel obstruction were reported. Other complications such as seroma, entero-cutaneous fistula and postoperative fever were also documented. For each patient, postoperative complications were graded individually according to the Clavien-Dindo classification of surgical complications (11). Readmission rate was calculated by considering the number of patients who were readmitted in the first 30 days after discharge for a cause related to the intervention, and calculating the ratio between the total number of patients in each group.

All patients received the same antibiotic treatment, according to our institution's protocol, in consensus with the Preventive Medicine Service. Preoperatively, a single dose of Amoxicillin-Clavulanic acid (40 mg $/ \mathrm{kg}$, maximum dose 2 grams) was administered to all patients. After surgery, the postoperative antibiotic was only continued in gangrenous appendicitis (Amoxicillin-Clavulanic Acid for 5 days), and in appendicitis complicated by peritonitis or appendicular mass (Gentamicin, Metronidazole and Amoxicillin-Clavulanic Acid for 7 days). In patients who underwent surgery after March 9, 2020 (COVID-19 group), patients were screened for SARS-CoV-2 infection before surgery. A swab was obtained from the 
nasopharyngeal mucosa, in which the virus genome was identified by real-time polymerase chain reaction test (12). The study protocol conformed to the guidelines of the 1975 Declaration of Helsinki and was approved by our institutional review board. Because of the nature of the aggregate data, the retrospective design of this study, as well as the importance of sharing research results and filling knowledge gaps, the institutional review board waived ethical approval.

Data were collected in Microsoft Excel software version 2010 (Redmond, WA, EE.UU.), and analyzed with SPSS Statistic version 22 (Chicago, IL, USA). Kolmogorov-Smirnoff and Shapiro-Wilk tests were used to compare the distributions of numerical variables. Continuous variables normally distributed were expressed as mean and standard deviation (SD), and were analyzed by Student t-test of independent samples. To analyze continuous data not normally distributed, Mann-Whitney test was used, and were expressed as median and interquartile range (Q1-Q3). Categorical variables were expressed as frequency (n) and percentage (\%). and were analyzed by Chi square test, or Fisher's test when the first one could not be applied. Odds ratios (OR) were calculated with $95 \%$ confidence intervals. All statistical calculations were performed with two tails and the statistical significance was established with a value of $p<0.05$.

\section{Results}

A total of 1274 patients were included (797 males and 477 females), with a median age at diagnosis of $10.3 \pm 3.3$ years, of whom 288 were operated on after March 9, 2020 (COVID-19 group) and 986 before that date (control group), with no demographic differences between them. COVID-19 group patients presented generalized abdominal pain on arrival at the emergency department in $41.3 \%$ of cases, which was significantly higher than those in the control group $(18.4 \% ; p<0.001)$. On the contrary, in the control group, abdominal pain located in the right iliac fossa (RIF) was observed in $81.6 \%$ of the patients, compared to $58.7 \%$ in the COVID-19 group. Time from symptom onset was significantly longer in COVID19 group patients ( 34.5 vs. 24.2 hours; $p=0.021$ ), although no differences in associated symptoms were observed between both groups. Table 1 describes demographic features, associated symptoms, and time since onset of symptoms in both groups. 
Table 1

Demographic features, associated symptoms, and time since onset of symptoms in both groups.

\begin{tabular}{|c|c|c|c|}
\hline & $\begin{array}{l}\text { COVID-19 group } \\
(n=288)\end{array}$ & $\begin{array}{l}\text { Control group } \\
(n=986)\end{array}$ & p-Value \\
\hline Gender, n(\%) & $194(67.4)$ & $603(61.2)$ & 0.056 \\
\hline - Males & $94(32.6)$ & $383(38.8)$ & \\
\hline \multicolumn{4}{|l|}{ - Females } \\
\hline Age (years); mean (SD) & $10.0(3.1)$ & $10.4(3.4)$ & 0.222 \\
\hline BMI (Kg/m2); media (SD) & $18.4(3.8)$ & $18.9(6.4)$ & 0.393 \\
\hline Abdominal pain & $169(58.7)$ & $805(81.6)$ & $<0.001$ \\
\hline - Located in RIF & $119(41.3)$ & $181(18.4)$ & \\
\hline \multicolumn{4}{|l|}{ - Generalized } \\
\hline Associated symptoms; n(\%) & $118(41)$ & $375(38)$ & 0.405 \\
\hline - Fever & $190(66)$ & $663(67.2)$ & 0.687 \\
\hline - Nausea & $167(58)$ & $576(58.4)$ & 0.896 \\
\hline - Vomiting & $37(12.8)$ & $123(12.5)$ & 0.867 \\
\hline - Diarrhea & $23(8)$ & $65(6.6)$ & 0.412 \\
\hline - Constipation & $24(8.3)$ & $72(7.3)$ & 0.566 \\
\hline - Dysuria & $119(41.3)$ & $371(37.7)$ & 0.262 \\
\hline - Anorexia & $15(5.2)$ & $61(6.2)$ & 0.538 \\
\hline \multicolumn{4}{|l|}{ - Respiratory symptoms } \\
\hline Time from symptoms onset (hours); median (Q1-Q3) & $34(24-48)$ & $24(8-36)$ & 0.021 \\
\hline
\end{tabular}

Regarding intraoperative findings, COVID-19 group patients presented a higher percentage of complicated AA when compared to the control group (20.1\% vs. $14 \%$; OR: 1.55 ; Cl $95 \%$ [1.10-2.18]; $p=0.008)$. These patients also had a longer hospital stay ( 3.5 vs. 2.8 days; $p=0.042)$ as well as a higher rate of postoperative complications (21.5\% vs. $15.7 \%$; OR: 1.47 ; Cl 95\% [(1.06-2.04)]; $p=0.008)$ when compared to the control group. When analyzing postoperative complications, we found that surgical wound infections, intra-abdominal abscesses and intestinal obstructions were more frequent in the COVID-19 group, although this difference was only statistically significantly different in the case of surgical wound infections ( $6.9 \%$ vs. $4.1 \% ; p=0.042)$. Furthermore, when analysing complications according to the Clavien-Dindo classification, patients in the COVID-19 group had a statistically higher percentage of grade III complications compared to the control group ( $37.1 \%$ vs. $24 \%$; $p 0.001)$. Readmission rate was $4.9 \%$ in the COVID-19 group vs. $3.1 \%$ in the control group, this difference not being statistically significant. 
Table 2 compares the intraoperative findings, hospital stay, postoperative complications, severity of complications according to the Clavien-Dindo classification and readmission rate between the two groups. 
Table 2

Comparison of intraoperative findings, hospital stay, postoperative complications and readmission rate between the two groups. OR, odds ratio. P-value $<0.05$ were accepted as significant.

\begin{tabular}{|c|c|c|c|c|}
\hline & $\begin{array}{l}\text { COVID-19 } \\
\text { group }(n=288)\end{array}$ & $\begin{array}{l}\text { Control } \\
\text { group } \\
(n= \\
986)\end{array}$ & $\begin{array}{l}\mathrm{p}- \\
\text { Value }\end{array}$ & $\begin{array}{l}\text { OR (Cl } \\
95 \%)\end{array}$ \\
\hline $\begin{array}{l}\text { Type acute appendicitis, } \mathrm{n}(\%) \\
\text { - Uncomplicated } \\
\text { - Complicated }\end{array}$ & $\begin{array}{l}230(79.9) \\
58(20.1)\end{array}$ & $\begin{array}{l}848 \\
(86) \\
138 \\
(14)\end{array}$ & 0.008 & $\begin{array}{l}1.55 \\
(1.10- \\
2.18)\end{array}$ \\
\hline Mean hospital stay, mean (SD), days & $3.5(2.4)$ & $\begin{array}{l}2.8 \\
(2.1)\end{array}$ & 0.042 & - \\
\hline $\begin{array}{l}\text { Postoperative complications; n(\%) } \\
\text { - Surgical wound infection } \\
\text { - Surgical wound dehiscence } \\
\text { - Intra-abdominal abscess } \\
\text { - Bowel obstruction }\end{array}$ & $\begin{array}{l}62(21.5) \\
20(6.9) \\
6(2.1) \\
27(9.4) \\
5(1.7)\end{array}$ & $\begin{array}{l}154 \\
(15.6) \\
37(4.1) \\
30(3.1) \\
64(6.5) \\
12(1.2)\end{array}$ & $\begin{array}{l}0.021 \\
0.042 \\
0.346 \\
0.095 \\
0.499\end{array}$ & $\begin{array}{l}1.47 \\
(1.06- \\
2.04) \\
1.76 \\
(1.01- \\
3.07) \\
0.66 \\
(0.27- \\
1.59) \\
1.49 \\
(0.93- \\
2.39) \\
1.43 \\
(0.50- \\
4.10)\end{array}$ \\
\hline $\begin{array}{l}\text { Other, n(\%) } \\
\text { - Seroma } \\
\text { - Postoperative fever } \\
\text { - Enterocutaneous fistula }\end{array}$ & $\begin{array}{l}3(1) \\
1(0.3) \\
-\end{array}$ & $\begin{array}{l}6(0.6) \\
4(0.4) \\
1(0.1)\end{array}$ & & $\begin{array}{l}- \\
-\end{array}$ \\
\hline $\begin{array}{l}\text { Postoperative complications according to } \\
\text { Clavien-Dindo classification; } \mathrm{n}(\%) \\
\text { - Grade I } \\
\text { - Grade II } \\
\text { - Grade III }\end{array}$ & $\begin{array}{l}19(30.6) \\
20(32.3) \\
23(37.1)\end{array}$ & $\begin{array}{l}65 \\
(42.2) \\
52 \\
(33.8) \\
37 \\
(24.0)\end{array}$ & 0.001 & $\begin{array}{l}- \\
-\end{array}$ \\
\hline Readmission rate; $\mathrm{n}(\%)$ & $14(4.9)$ & $31(3.1)$ & 0.164 & $\begin{array}{l}1.57 \\
(0.83- \\
3.0)\end{array}$ \\
\hline
\end{tabular}




\section{Discussion}

The present study demonstrates the negative mid-term effects in paediatric patients with AA during the first 9 months from the onset of the COVID-19 pandemic. After analysing 1274 cases a longer delay to first consultation or the ED, a higher rate of complicated appendicitis, a prolonged hospital stay and a higher postoperative complications rate in patients presenting during the COVID-19 pandemic period when compared with previous years has been observed.

Acute appendicitis is one of the most commonly reported causes of acute abdomen in children presenting to the ED, and it is traditionally believed to develop secondary to luminal obstruction either by fecalith or lymphoid hyperplasia (13). Progression of appendiceal distention to frank perforation occurs in $20-75 \%$ of cases and can impart significant morbidity (14). Yet, it is difficult to differentiate it from other causes as less than $50 \%$ of patients present with classic symptoms (15). Delay in presentation is a major driver of perforation, with perforation rates increasing linearly with duration of symptoms (16).

Since the onset of SARS-Cov-2 pandemic, management of acute appendicitis has been affected by epidemiological changes reported by multiple authors, with a delay the time of diagnosis, increased frequently peritonitis and more severe septic abdominal diseases (17). In this context, abdominal CT scans performed on patients with AA during the acute pandemic period show a higher severity of disease when compared with cases in the nonpandemic comparison period (18). These findings were also observed in patients at our centre, where we report an increase in complicated appendicitis and postoperative complications during the pandemic outbreak when compared with patients operated on during the same time period during the previous years (9). We found as a possible explanation changes in parental behaviour during the COVID-19 outbreak, where many parents described a delay in seeking assessment for their child's abdominal pain due to fears of in-hospital SARS-CoV-2. Many other studies in different disciplines have confirmed a significant reduction of ED visits during the COVID-19 outbreak, which raises concern about the effect of this behavior on patients' prognoses (4). Furthermore, in the areas most affected by the pandemic such as our region, these concerns dramatically influenced people's willingness to seek medical care, even to the point of avoiding acute care. Mantica et al. found that the lowest numbers of ED visits corresponded with the highest numbers of deaths from COVID-19 (19).

On this background, once the first wave of the pandemic was over, and once the home confinement measures were lifted, in our centre we continued observing the same tendency of delay in going to the ED in children with abdominal pain, as well as a high proportion of complicated appendicitis, so we decided to analyse the results of patients operated on during the first 9 months after the beginning of the pandemic. According to the results of our study, although patients presented to the ED with comparable individual demographic features in both groups, the cohort of patients who underwent surgery after the COVID-19 outbreak presented a difference of more than 10 hours in the time from onset of symptoms (34 vs. 24 hours). This delay in diagnosis and early preoperative antibiotic treatment was directly related to the increase in complicated appendicitis in this group of patients when compared to the control group. In addition, COVID-19 group patients exhibited an almost 1.5 times higher rate of surgical complications 
( $21.5 \%$ vs. $15.6 \%)$, with increased severity values in the Claviden-Dindo classification. As an expected consequence, the length of hospitalization was significantly increased in the COVID-19 group. These findings illustrate a critical scenario that began in the pandemic outbreak in March 2020, and which has been sustained over time during the first 9 months of the pandemic: throughout a period in which healthcare resources and funds have been concentrated on the pandemic (COVID-19 screening tests, clinical trials vaccination studies), an increased burden of surgical complications has increased both hospital costs and assistance efforts in children with acute appendicitis.

During the home confinement period in Europe the rate of perforated appendicitis in childhood and adolescence increased significantly. Potentially this was attributed to a delayed presentation to the ED due to the "stay-at home" policy, parent's fear of the hospital environment or the wish not to strain hospital workload further with seemingly manageable conditions. However, after the end of the COVID-19 outbreak and the end of the curfew period the results of our study continue revealing negative consequences derived from the delay in attending the ED of parents with children with abdominal pain. This diagnostic delay has again resulted in an increase in complicated appendicitis, a higher rate of postoperative complications and a longer hospital stay. This can no longer be justified by home confinement and mobility restriction measures, so we must look for other causes for these findings. One possible explanation for the reluctance of parents to go to the ED with their children may be the lack of available vaccines during 2020. At the beginning of 2021, the first national vaccination campaigns on the most at-risk population (health professionals, elderly patients) started. It will be necessary to assess the impact of these vaccination campaigns on parents' behaviour in relation to coming to the hospital setting. In addition, another fact that may explain the delay in diagnosis is that local health centres remain closed, with only telephone consultations. This may contribute to parents treating abdominal pain with analgesia at home, and only when it does not improve after several days do they decide to go to the ED. Therefore, reopening the health centres during 2021 may help to decrease the high rate of complicated appendicitis encountered during the first 9 months after the start of the pandemic. Because of potential long-term sequelae of complicated appendicitis such as intestinal adhesions with obstruction these adverse effects of a reduced utilization of health care facilities should be considered for future political decision making. It is important to advise legal authorities not to discourage the population to seek timely medical attention in case of emergency conditions in order to avoid collateral damage in near-future or on-going pandemic situations (20). Parents and general pediatricians practitioners should be aware of these problems to avoid a time delay from initial symptoms to consultation (21). Health authorities should encourage vaccination of the general population and facilitate access to health care. These measures will need to be evaluated in patients undergoing surgery for acute appendicitis in the coming months.

The retrospective and single centre design are some of the limitations in our study. Due to the restricted postoperative follow-up in the first 30-days after surgery, the study is also lacking in long-term follow-up and the effect of the children's recovery in terms of quality of life, and also the implications of absence from school and return to normal activity. Multicentre studies assessing the long-term consequences of the impact of pandemic COVID-19 in children with acute appendicitis are needed. 


\section{Conclusion}

This study shows the negative consequences of the COVID-19 pandemic during the first 9 months of the pandemic in children with AA. Unfortunately, the negative effects have been sustained during successive waves of infection, without being limited to the COVID-19 outbreak. The delay in attending the ED and the higher rate of complicated appendicitis associated with the longer evolution time of appendicitis have led to a higher rate of postoperative complications and a longer hospital stay when compared to children operated on before the onset of the pandemic.

\section{References}

1. [Spanish G (2020) Real Decreto $463 / 2020$, de 14 de marzo, por el que se declara el estado de alarma para la gestión de la situación de crisis sanitaria ocasionada por el COVID-19.

https://boe.es/boe/dias/2020/03/11/pdfs/BOE-A-2020-3434.pdfs/BOE-A-2020-3434.pdf. [Accessed 10 May 2021; in Spanish]]

2. Centers for Disease Control and Prevention (CDC). What to do if you are sick (2020) https://www.cdc.gov/coronavirus/2019-ncov/if-you-are-sick/steps-when-sick.html. [Accessed 15 May 2021]

3. Keesara S, Jonas A, Schulman K (2020) Covid-19 and Health Care's Digital Revolution. N Engl J Med 382(23):e82

4. Lazzerini M, Barbi E, Apicella A, Marchetti F, Cardinale F, Trobia G (2020) Delayed access or provision of care in Italy resulting from fear of COVID-19. Lancet Child Adolesc Health 4:e10-e11

5. Perez KS, Allen SR (2018) Complicated appendicitis and considerations for interval appendectomy. Journal of the American Academy of Physician Assistants 31:35-41

6. Orthopoulos G, Santone E, Izzo F, Tirabassi M, Pérez-Caraballo AM, Corriveau N, Jabbour N (2021) Increasing incidence of complicated appendicitis during COVID-19 pandemic. Am J Surg 221:10561060

7. Gao Z, Li M, Zhou H, Liang Y, Zheng C, Li S et al (2020) Complicated appendicitis are common during the epidemic period of 2019 novel coronavirus (2019-nCoV). Asian J Surg 43:1002-1005

8. Wichmann D, Schweizer U, Wulff D, Thiel K, Beltzer C, Königsrainer A, Archid R. Incidence of Perforated Appendicitis during the COVID-19 Pandemic: Lessons to Be Considered in the Second Wave. J Gastrointest Surg. 2021 Feb 8:1-3

9. Delgado-Miguel C, Muñoz-Serrano AJ, Miguel-Ferrero M, De Ceano-Vivas M, Calvo C, Martínez L (2021) Complicated Acute Appendicitis during COVID-19 Pandemic: The Hidden Epidemic in Children. Eur J Pediatr Surg. Feb 22

10. Berríos-Torres SI, Umscheid CA, Bratzler DW, Leas B, Stone EC, Kelz RR et al (2017) Centers for Disease Control and Prevention Guideline for the Prevention of Surgical Site Infection, 2017. JAMA Surg 152:784-791 
11. Dindo D, Demartines N, Clavien PA (2004) Classification of surgical complications: a new proposal with evaluation in a cohort of 6336 patients and results of a survey. Ann Surg 240:205-213

12. Corman VM, Landt O, Kaiser M, Molenkamp R, Meijer A, Chu DK et al (2020) Detection of 2019 novel coronavirus (2019-nCoV) by real-time RT-PCR. Euro Surveill 25:2000045

13. Fisher JC, Tomita SS, Ginsburg HB, Gordon A, Walker D, Kuenzler KA (2021) Increase in Pediatric Perforated Appendicitis in the New York City Metropolitan Region at the Epicenter of the COVID-19 Outbreak. Ann Surg 273:410-415

14. Bhangu A, Soreide K, Di Saverio S et al (2015) Acute appendicitis: modern understanding of pathogenesis, diagnosis, and management. Lancet 386:1278-1287

15. Alsuwailem AB, Turkistani R, Alomari M (2020) Complicated Appendicitis in a Pediatric Patient With COVID-19: A Case Report. Cureus 12:e8677

16. Papandria D, Goldstein SD, Rhee D et al (2013) Risk of perforation increases with delay in recognition and surgery for acute appendicitis. J Surg Res 184:723-729

17. Reichert M, Sartelli M, Weigand MA, Doppstadt C, Hecker M, Reinisch-Liese A et al (2020) WSES COVID-19 emergency surgery survey collaboration group. Impact of the SARS-CoV-2 pandemic on emergency surgery services-a multi-national survey among WSES members. World J Emerg Surg 15:64

18. Romero J, Valencia S, Guerrero A (2020) Acute Appendicitis During Coronavirus Disease 2019 (COVID-19): Changes in Clinical Presentation and CT Findings. J Am Coll Radiol 17:1011-1013

19. Mantica G, Riccardi N, Terrone C, Gratarola A (2020) Non-COVID-19 visits to emergency departments during the pandemic: the impact of fear. Public Health 183:40-41

20. Schäfer FM, Meyer J, Kellnar S, Warmbrunn J, Schuster T, Simon S et al (2021) Increased Incidence of Perforated Appendicitis in Children During COVID-19 Pandemic in a Bavarian Multi-Center Study. Front Pediatr 9:683607

21. Burgard M, Cherbanyk F, Nassiopoulos K, Malekzadeh S, Pugin F, Egger B (2021) An effect of the COVID-19 pandemic: Significantly more complicated appendicitis due to delayed presentation of patients! PLoS One 16(5):e0249171 